\title{
ON PARTICLE-IN-CELL APPROXIMATIONS TO PARTICLE-IN-FOURIER SCHEMES
}

\author{
${ }^{1}$ Max-Planck-Institut für Plasmaphysik \\ Boltzmannstr. 2, D-85748 Garching, Germany \\ 2 Technische Universität München, Zentrum Mathematik \\ Boltzmannstr. 3, D-85748 Garching, Germany \\ jakob.ameres@gmail.com \\ martin.campos-pinto@ipp.mpg.de \\ katharina.kormann@ipp.mpg.de
}

JAKOB AMERES ${ }^{2,1}$, MARTIN CAMPOS PINTO ${ }^{1}$ AND KATHARINA KORMANN ${ }^{1,2}$

Key words: Spectral Particle-In-Cell, Particle-In-Fourier, anti-aliasing, nonequispaced Fast Fourier transform, Vlasov-Maxwell

\begin{abstract}
The gridless Particle-In-Fourier method where particles are directly deposited onto a Fourier basis features excellent energy and momentum conservation properties, but is computationally expensive due to the global nature of its basis functions. In this paper, we investigate analytically and numerically how well a back-filtered spectral Particle-In-Cell method can approximate the Particle-In-Fourier method. The study is extended to a recent variational spectral PIC method which has a discrete Hamiltonian structure.
\end{abstract}

\section{Introduction}

In a recent article [2], a general framework was proposed for spectral electromagnetic particle schemes that preserve certain key properties such as Gauss' laws, the total momentum, or a discrete Hamiltonian structure. A particular feature of this framework is that it encompasses both the Particle-in-Fourier (PIF) method $[7,1]$ which is based on a gridless particle-field coupling, and spectral Particle-in-Cell (PIC) methods [5] where the coupling is done through an DFT grid.

In this article we investigate how well spectral PIC schemes may approximate gridless simulations, provided a proper back-filtering is used in Fourier space as suggested in [6, 2]. In Section 2 we recall the main steps of both methods and revisit a classical a priori error estimate in Section 3. This estimate is then assessed numerically by considering errors in individual modes in Section 4, and global errors in the electromagnetic field energy in Section 5. In Section 6 we finally study the spectral fidelity of the different solvers in a single-mode electrostatic oscillation. This allows to verify the anti-aliasing properties of smoothed particle shapes in spectral PIC methods, and to highlight the superior accuracy of back-filtered PIC schemes. 


\section{Particle-in-Fourier and spectral PIC methods}

A common feature of spectral particle methods is to solve Maxwell's equations in Fourier space

$$
\left\{\begin{aligned}
-\frac{\mathrm{d}}{\mathrm{d} t} \boldsymbol{E}_{\boldsymbol{k}}+\left(\frac{2 \mathrm{i} \pi \boldsymbol{k}}{L}\right) \times \boldsymbol{B}_{\boldsymbol{k}}=\boldsymbol{J}_{\boldsymbol{k}}^{S}, & \\
\frac{\mathrm{d}}{\mathrm{d} t} \boldsymbol{B}_{\boldsymbol{k}}+\left(\frac{2 \mathrm{i} \pi \boldsymbol{k}}{L}\right) \times \boldsymbol{E}_{\boldsymbol{k}}=0 & \text { for } \quad \boldsymbol{k} \in \llbracket-K, K \rrbracket^{3}
\end{aligned}\right.
$$

and to push the $N$ particles along trajectory equations of the form

$$
\left\{\begin{array}{l}
\frac{\mathrm{d}}{\mathrm{d} t} \boldsymbol{X}_{p}=\boldsymbol{V}_{p}, \\
\frac{\mathrm{d}}{\mathrm{d} t} \boldsymbol{V}_{p}=\frac{q_{p}}{m_{p}}\left(\boldsymbol{E}^{S}\left(\boldsymbol{X}_{p}\right)+\boldsymbol{V}_{p} \times \boldsymbol{B}^{S}\left(\boldsymbol{X}_{p}\right)\right) \quad \text { for } \quad p \in \llbracket 1, N \rrbracket .
\end{array}\right.
$$

Here, the coupling current coefficients $\boldsymbol{J}_{\boldsymbol{k}}^{S}$ are obtained from the particle current

$$
J_{N}(t, x):=\sum_{p=1}^{N} q_{p} V_{p}(t) \delta\left(x-\boldsymbol{X}_{p}(t)\right),
$$

and the coupling fields $\boldsymbol{E}^{S}$ and $\boldsymbol{B}^{S}$ are obtained from the spectral fields

$$
E_{K}(t, x):=\sum_{k \in \llbracket-K, K \rrbracket^{3}} E_{k}(t) \mathrm{e}^{\frac{2 i \pi k \cdot x}{L}} \text { and } B_{K}(t, x):=\sum_{k \in \llbracket-K, K \rrbracket^{3}} B_{k}(t) \mathrm{e}^{\frac{2 i \pi k \cdot x}{L}} \text {. }
$$
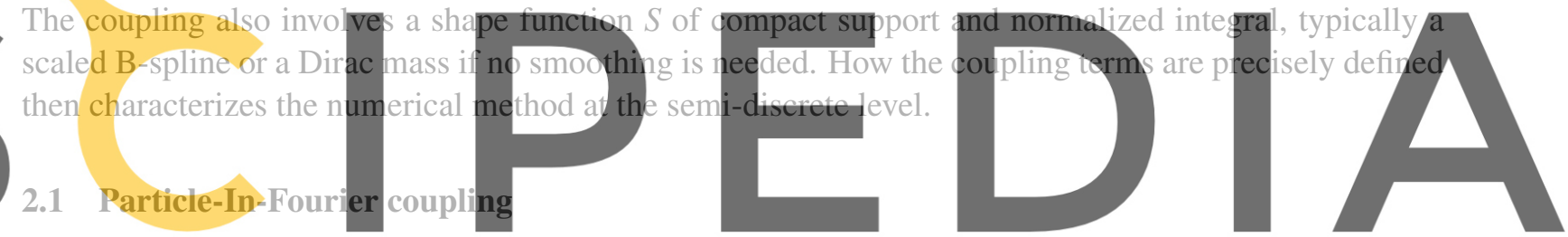

In the gridless Particle-In-Fourier method, the coupling current terms are given by the Fourier coeffi-

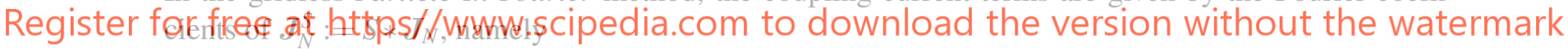

$$
\boldsymbol{J}_{\boldsymbol{k}}^{S}:=\mathcal{F}_{\boldsymbol{k}}\left(S * \boldsymbol{J}_{N}\right)=\sigma_{\boldsymbol{k}} \mathcal{F}_{\boldsymbol{k}}\left(\boldsymbol{J}_{N}\right)=\sigma_{\boldsymbol{k}} \sum_{p=1 \cdots N} q_{p} \boldsymbol{V}_{p}\left(\frac{1}{L}\right)^{3} \mathrm{e}^{-\frac{2 \mathrm{i} \pi k \cdot X_{p}}{L}},
$$

where we have set $\sigma_{k}:=L^{3} \mathcal{F}_{k}(S)$, and denoted by

$$
\mathcal{F}_{\boldsymbol{k}}(G):=\left(\frac{1}{L}\right)^{3} \int_{[0, L]^{3}} G(\boldsymbol{x}) \mathrm{e}^{-\frac{2 i \pi k \cdot \boldsymbol{x}}{L}} \mathrm{~d} \boldsymbol{x}
$$

the $\boldsymbol{k}$-th (continuous) Fourier coefficient of a function $G$. The coupling fields are then defined as

$$
\left\{\begin{array}{l}
\boldsymbol{E}^{S}\left(\boldsymbol{X}_{p}\right)=\int_{[0, L]^{3}} \boldsymbol{E}_{K}(\boldsymbol{x}) S\left(\boldsymbol{x}-\boldsymbol{X}_{p}\right) \mathrm{d} \boldsymbol{x}=L^{3} \sum_{\boldsymbol{k} \in \llbracket-K, K \rrbracket^{3}} \overline{\sigma_{\boldsymbol{k}}} \boldsymbol{E}_{\boldsymbol{k}} \mathrm{e}^{\frac{2 \mathrm{i} \pi \boldsymbol{k} \cdot \boldsymbol{X}_{p}}{L}} \\
\boldsymbol{B}^{S}\left(\boldsymbol{X}_{p}\right)=\int_{[0, L]^{3}} \boldsymbol{B}_{K}(\boldsymbol{x}) S\left(\boldsymbol{x}-\boldsymbol{X}_{p}\right) \mathrm{d} \boldsymbol{x}=L^{3} \sum_{\boldsymbol{k} \in \llbracket-K, K \rrbracket^{3}} \overline{\sigma_{\boldsymbol{k}}} \boldsymbol{B}_{\boldsymbol{k}} \mathrm{e}^{\frac{2 \mathrm{i} \pi \boldsymbol{k} \cdot \boldsymbol{X}_{p}}{L}} .
\end{array}\right.
$$

Here we note that the particle shape $S$ essentially amounts in a specific Fourier filtering in the coupling terms. In particular for a Dirac shape $S=\delta$ we have $\sigma_{k}=1$ for all $\boldsymbol{k} \in \mathbb{Z}^{3}$. 


\subsection{Particle-In-Cell coupling with smooth shapes}

In spectral PIC methods, the coupling involves a grid (of $M$ cells) where the continuous Fourier coefficients (6) are approximated by discrete ones,

$$
\mathcal{F}_{M, k}(G):=\left(\frac{1}{M}\right)^{3} \sum_{\boldsymbol{m} \in \llbracket 1, M \rrbracket^{3}} G(\boldsymbol{m} h) \mathrm{e}^{-\frac{2 i \pi k \cdot m}{M}} \quad \text { with } \quad h=\frac{L}{M} .
$$

The current source terms are then given as

$$
\boldsymbol{J}_{\boldsymbol{k}}^{S}:=\mathcal{F}_{M, \boldsymbol{k}}\left(S * \boldsymbol{J}_{N}\right)
$$

which amounts to first depositing this current on the grid,

$$
\boldsymbol{J}_{m}^{\text {pic }}:=\left(S * \boldsymbol{J}_{N}\right)(\boldsymbol{m} h)=\sum_{p=1 \ldots N} a_{p} \boldsymbol{V}_{p} S\left(\boldsymbol{m} h-\boldsymbol{X}_{p}\right) \quad \text { for } \boldsymbol{m} \in \llbracket 1, M \rrbracket^{3}
$$

and then performing a Discrete Fourier Transform (DFT),

$$
J_{k}^{S}=\left(\frac{1}{M}\right)^{3} \sum_{m \in \llbracket 1, M \rrbracket^{3}} J_{m}^{\mathrm{pic}} \mathrm{e}^{-\frac{2 \mathrm{i} \pi k \cdot m}{M}} \quad \text { for } k \in \llbracket-K, K \rrbracket^{3} .
$$

The pushing fields are defined by a discrete convolution,
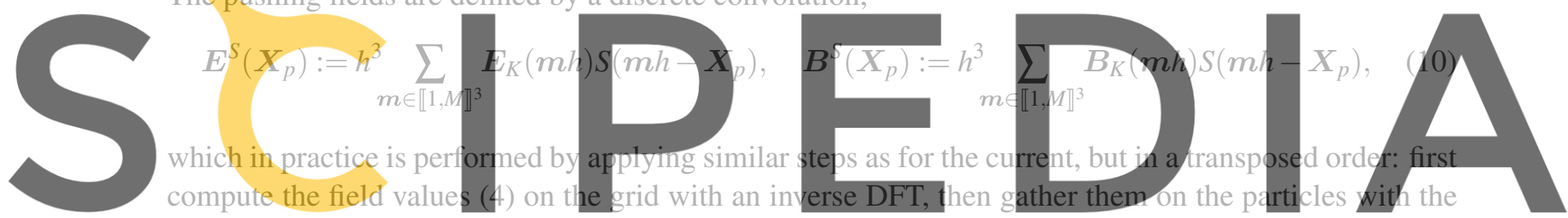

shape function $S$.

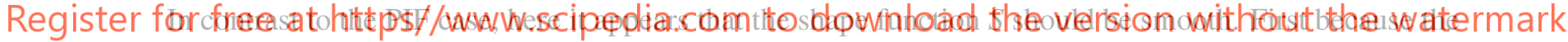
above coupling is only well-defined for a continuous shape $S$, and more importantly because of the antialiasing properties of smooth shape functions. Indeed, DFT is inherently associated with aliasing errors, due to high-frequency modes of the continuous source that get folded into the computational range. As they amount in a low-pass filtering of the source before the DFT is applied, smooth shapes reduce these high frequency modes and hence the aliasing errors.

A common choice is to use a (periodic) B-spline of degree $\kappa \geq 1$ scaled to the grid,

$$
S(\boldsymbol{x}):=\sum_{\boldsymbol{r} \in \mathbb{Z}^{3}} S_{\kappa}^{h}(\boldsymbol{x}+\boldsymbol{r} L) \quad \text { where } \quad S_{\kappa}^{h}(\boldsymbol{x}):=\left(\frac{1}{h}\right)^{3} \prod_{\alpha \in \llbracket 1,3 \rrbracket} \hat{S}_{\kappa}\left(\frac{x_{\alpha}}{h}\right),
$$

with cardinal univariate B-splines defined as $\hat{S}_{0}(x):=\mathbb{1}_{\left[-\frac{1}{2}, \frac{1}{2}\right]}(x)$ and $\hat{S}_{\mathrm{K}}(x):==\int_{-\frac{1}{2}}^{\frac{1}{2}} \hat{S}_{\mathrm{K}-1}(x-y) \mathrm{d} y$ for $\kappa \geq 1$. Then the convolution by $S$ amounts to a filtering in Fourier space with coefficients

$$
\sigma_{k}:=L^{3} \mathcal{F}_{k}(S)=\prod_{\alpha=1}^{3}\left(\operatorname{sinc}\left(\frac{\pi k_{\alpha}}{M}\right)\right)^{\mathrm{K}+1} \quad \text { where } \quad \operatorname{sinc}(\theta):=\frac{1}{\theta} \sin \theta .
$$




\subsection{Variational Fourier-GEMPIC scheme and back-filtering techniques}

In [2] the PIC coupling has been revisited and a new method has been derived from a variational principle based on a discrete Lagrangian functional for the Vlasov-Maxwell system. In this derivation the particle-field coupling is described by projection operators on the discrete Fourier spaces, that commute with the differential operators involved in the de Rham sequence. As a consequence of this variational derivation, the resulting Fourier-GEMPIC method possess a discrete Hamiltonian structure and preserves the discrete Gauss laws associated with the de Rham commuting diagram. It may thus be seen as a spectral extension to the GEMPIC scheme [4] based on spline Finite Elements and point particle shapes. The standard PIC coupling described above can be seen as a variant which is not variational but preserves the total momentum.

An interesting feature of the variational derivation (and its momentum-preserving variant) is that it allows general Fourier filters in the coupling terms. For the gridless PIF method where the coupling (5)-(7) reads

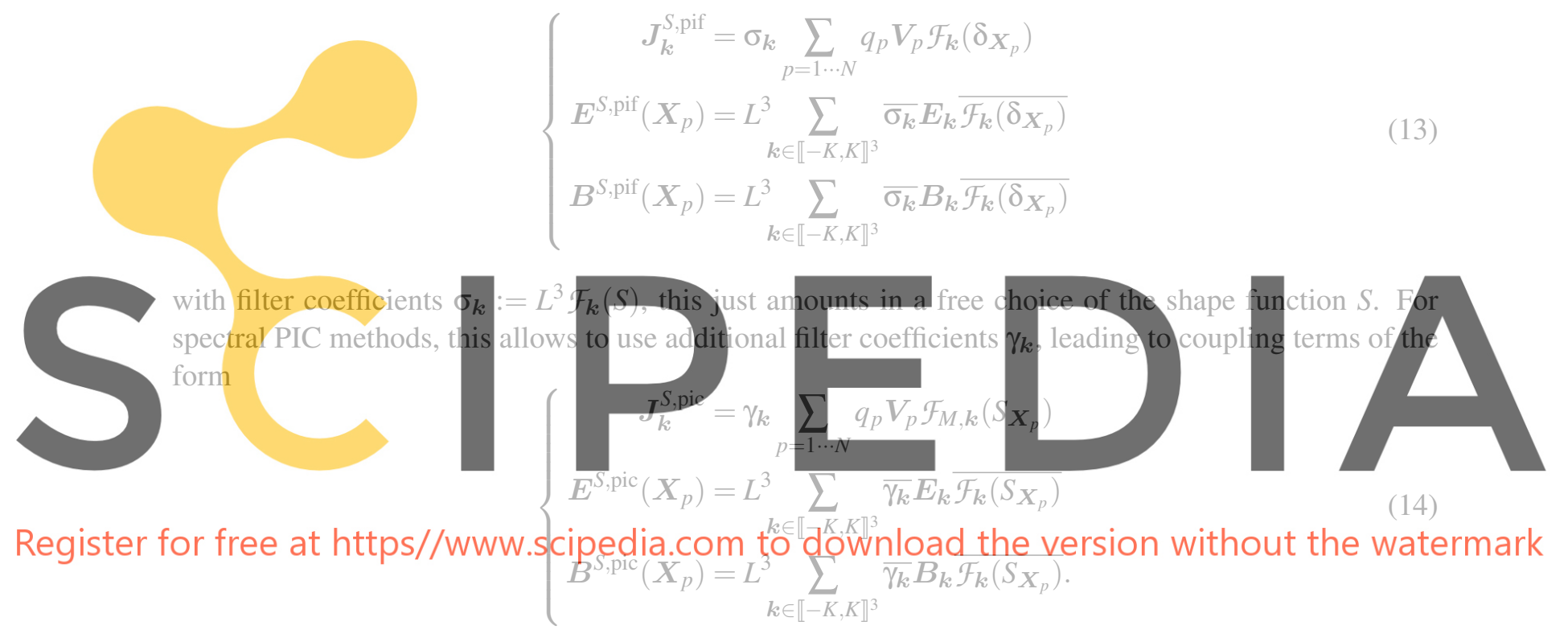

Here the situation is quite different, because as was previously recalled, the shape functions are used before the DFT, whereas the $\gamma$-filtering is used afterwards. In particular, this opens the possibility of using the $\gamma$-filter as a back-filtering technique to compensate for the smooth shape functions $S$. The motivation for such a strategy is twofold:

- First, it allows to revisit the (back-filtered) PIC coupling as a high-order approximations to the gridless PIF coupling.

- Second, it leads to a better modelling of the high modes within the computational range, despite the smoothing effect of the particle shapes.

In the next sections, we will validate these properties by a priori estimates and numerical experiments. Since the antialiasing and back-filtering techniques can also be applied on the variational Fourier-GEMPIC method, we chose to perform this study on this latter method. We note, however, that similar results were obtained with the momentum-preserving scheme. For lack of space we do not report them here. 


\section{A priori error estimate}

In this section we present an a-priori error estimate for the approximation of continuous Fourier coefficients by discrete ones. This estimate essentially follows similar results established in [9], see also [8], where this approximation procedure is described as a Nonequispaced Fast Fourier Transform. We state it for univariate shape functions, as the generalization to tensor-product ones is straightforward.

Lemma 1 Let $S^{\text {pif }}$ be an arbitrary shape, and let $S^{\text {pic }}$ be a periodic B-spline (11) of degree $\kappa \geq 1$ and step-size $h=\frac{L}{M}$. Then the discrete Fourier transform of $S_{X_{p}}^{\mathrm{pic}}(x)=S^{\mathrm{pic}}\left(x-X_{p}\right)$ with filter coefficients

$$
\gamma_{k}:=\frac{\mathcal{F}_{k}\left(S^{\mathrm{pif}}\right)}{\mathcal{F}_{k}\left(S^{\mathrm{pic}}\right)}=L \mathcal{F}_{k}\left(S^{\mathrm{pif}}\right)\left(\frac{\pi k / M}{\sin (\pi k / M)}\right)^{\mathrm{\kappa}+1} \quad \text { for } \quad|k| \leq K \leq \frac{M-1}{2}
$$

converges at order $\kappa+1$ to the continuous Fourier transform of $S_{X_{p}}^{\mathrm{pif}}(x)=S^{\mathrm{pif}}\left(x-X_{p}\right)$. Specifically,

$$
\max _{|k| \leq K} \sup _{X_{p} \in[0, L]}\left|\mathcal{F}_{k}\left(S_{X_{p}}^{\mathrm{pif}}\right)-\gamma_{k} \mathcal{F}_{M, k}\left(S_{X_{p}}^{\mathrm{pic}}\right)\right| \leq 2\left\|\mathcal{F}\left(S^{\mathrm{pif}}\right)\right\|_{\ell^{\infty}}\left(\frac{K / M}{1-K / M}\right)^{\mathrm{K}+1}\left(1+\frac{1-K / M}{\kappa}\right)
$$

\section{holds for all $K \in \mathbb{N}$ and all $M \geq 2 K+1$, with $\left\|\mathcal{F}\left(S^{\text {pif }}\right)\right\|_{\ell^{\infty}}=\sup _{k \in \mathbb{Z}}\left|\mathcal{F}_{k}\left(S^{\text {pif }}\right)\right|$.}

Remark 1 This estimate indeed gives a $\mathrm{\kappa}+1$ convergence order, since we have
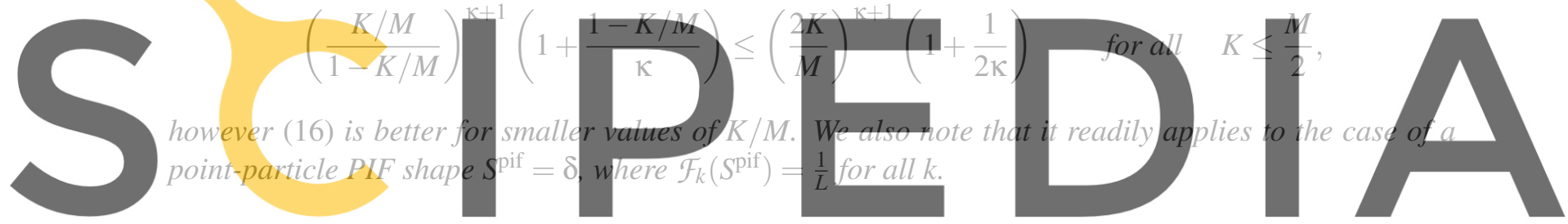

Proof. We recall and adapt the core estimate of [9, Th. 4.2]. Expressing the periodic B-spline (11) as a

Register forufree atithttps//www.scipedia.com to download the version without the watermark

$$
S(x)=\sum_{\ell \in \mathbb{Z}} c_{\ell} e^{\frac{2 i \pi}{L} \ell x} \quad \text { with } \quad c_{\ell}:=F_{\ell}(S)=\frac{1}{L}\left(\operatorname{sinc}\left(\frac{\pi \ell}{M}\right)\right)^{\kappa+1}
$$

we rewrite the discrete Fourier coefficients as

$$
\begin{aligned}
\mathcal{F}_{M, k}\left(S_{x_{p}}\right) & =\frac{1}{M} \sum_{m=0}^{M-1} S\left(m h-x_{p}\right) \mathrm{e}^{-\frac{2 i \pi}{L} k\left(m h-x_{p}\right)}=\mathrm{e}^{-\frac{2 i \pi}{L} k x_{p}} \frac{1}{M} \sum_{m=0}^{M-1} \sum_{\ell \in \mathbb{Z}} c_{\ell} \mathrm{e}^{\frac{2 i \pi}{L}(\ell-k)\left(m h-x_{p}\right)} \\
& =\mathrm{e}^{-\frac{2 i \pi}{L} k x_{p}} \frac{1}{M} \sum_{\ell \in \mathbb{Z}} c_{\ell} \mathrm{e}^{-\frac{2 i \pi}{L}(\ell-k) x_{p}} \sum_{m=0}^{M-1} \mathrm{e}^{\frac{2 i \pi}{M}(\ell-k) m}=\mathrm{e}^{-\frac{2 i \pi}{L} k x_{p}}\left(c_{k}+\sum_{r \in \mathbb{Z}^{*}} c_{k+r M} \mathrm{e}^{-\frac{2 i \pi}{L} r M x_{p}}\right)
\end{aligned}
$$

where we have used that $\sum_{m=0}^{M-1} \mathrm{e}^{\frac{2 i \pi}{M}(\ell-k) m}=M$ if $\ell-k \in M \mathbb{Z}$, and $=0$ otherwise. This formula essentially expresses the well-known aliasing phenomenon. As for the continuous Fourier coefficients, we have

$$
\mathcal{F}_{k}\left(S_{x_{p}}^{\mathrm{pif}}\right)=\frac{1}{L} \int_{0}^{L} S^{\mathrm{pif}}\left(x-x_{p}\right) \mathrm{e}^{-\frac{2 i \pi}{L} k x} \mathrm{~d} x=\mathcal{F}_{k}\left(S^{\mathrm{pif}}\right) \mathrm{e}^{-\frac{2 i \pi}{L} k x_{p}}=\gamma_{k} c_{k} \mathrm{e}^{-\frac{2 i \pi}{L} k x_{p}}
$$


which allows us to bound the error as

$$
\left|\mathcal{F}_{k}\left(S_{x_{p}}^{\mathrm{pif}}\right)-\gamma_{k} \mathcal{F}_{M, k}\left(S_{x_{p}}\right)\right|=\left|\gamma_{k} \mathrm{e}^{-\frac{2 i \pi}{L} k x_{p}} \sum_{r \in \mathbb{Z}^{*}} c_{k+r M} \mathrm{e}^{-\frac{2 i \pi}{L} r M x_{p}}\right| \leq\left|\mathcal{F}_{k}\left(S^{\mathrm{pif}}\right)\right| \sum_{r \in \mathbb{Z}^{*}}\left|\frac{c_{k+r M}}{c_{k}}\right| .
$$

Using that $\left|\frac{c_{k+r M}}{c_{k}}\right|=\left|\frac{k / M}{k / M+r}\right|^{\mathrm{\kappa}+1}$, we then estimate as in [9, Th. 4.2],

$$
\sum_{r \in \mathbb{Z}^{*}}\left|\frac{c_{k+r M}}{c_{k}}\right| \leq \frac{2}{L}\left(\frac{|k| / M}{1-|k| / M}\right)^{\kappa+1}\left(1+\frac{1-|k| / M}{\kappa}\right) \leq \frac{2}{L}\left(\frac{K / M}{1-K / M}\right)^{\kappa+1}\left(1+\frac{1-K / M}{\kappa}\right)
$$

which ends the proof.

\section{Numerical validation of the estimate}

We first perform a numerical verification of the error estimate (16) by using a set $\mathcal{X}$ of 10,000 particles distributed uniformly, on which we compare the PIF and PIC modes using a Dirac shape for the PIF coupling, $S^{\text {pif }}=\delta$, and a B-spline shape of degree $\kappa$ for the PIC coupling on a grid of size $M$. Thus, for a given mode $k \leq(M-1) / 2$, we compute the error

$$
\operatorname{error}(M, \kappa, k):=\max _{X_{p} \in X}\left|\mathcal{F}_{k}\left(\delta_{X_{p}}\right)-\gamma_{k} \mathcal{F}_{M, k}\left(S_{X_{p}}^{\mathrm{pic}}\right)\right|,
$$

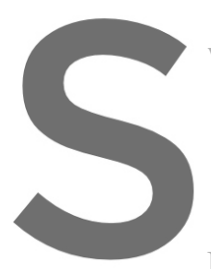

\section{with back-filtering coeffici

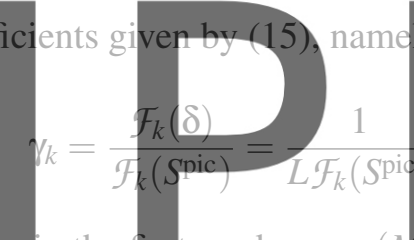

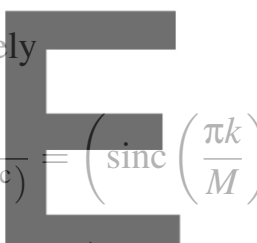
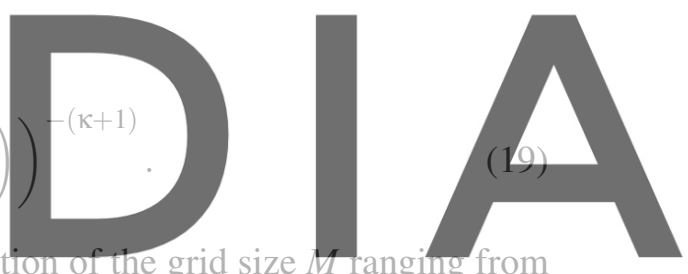

$2^{3}$ to $2^{14}$, for shape degrees $\mathrm{K}$ ranging from 1 to 7 . It can be seen that the predicted convergence rates

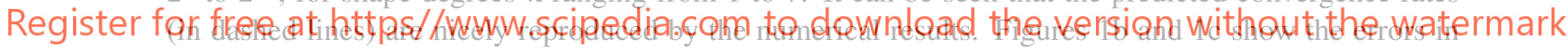

the second and third mode, i.e. error $(M, \kappa, 2)$ and $\operatorname{error}(M, \kappa, 3)$, respectively. For comparison, the errors

in the mode $k=1$ are shown in Figure $1 d$ for $\gamma_{k}=1$, that is when no back-filtering is used. It can be seen that in this case the convergence with respect to $M$ is of order two, independent of the degree for the shape function.

\section{Global convergence in the time evolution}

As Lemma 1 indicates that the PIC coupling (14) with proper back-filtering can be seen as a high order approximation of the PIF coupling (13), in this section we perform a numerical validation of this global convergence property. Specifically, we compare the results obtained with smoothed Fourier (sf) PIC schemes corresponding to $\gamma_{k}=1$, and back-filtered Fourier (bff) PIC schemes corresponding to (19). Again we note that the results shown here are for the variational GEMPIC method as explained in Section 2.3.

To do so we consider a reduced $1 \mathrm{~d} 2 \mathrm{v}$ model with the same Weibel and two-stream instablity test cases as in [2]. In this test, we restrict ourselves to the linear phase, i.e. we simulate these test cases over 200 


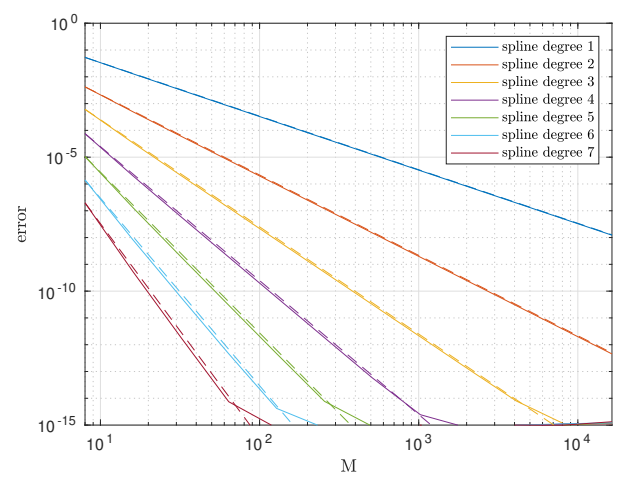

(a) $\operatorname{error}(M, \kappa, 1)$

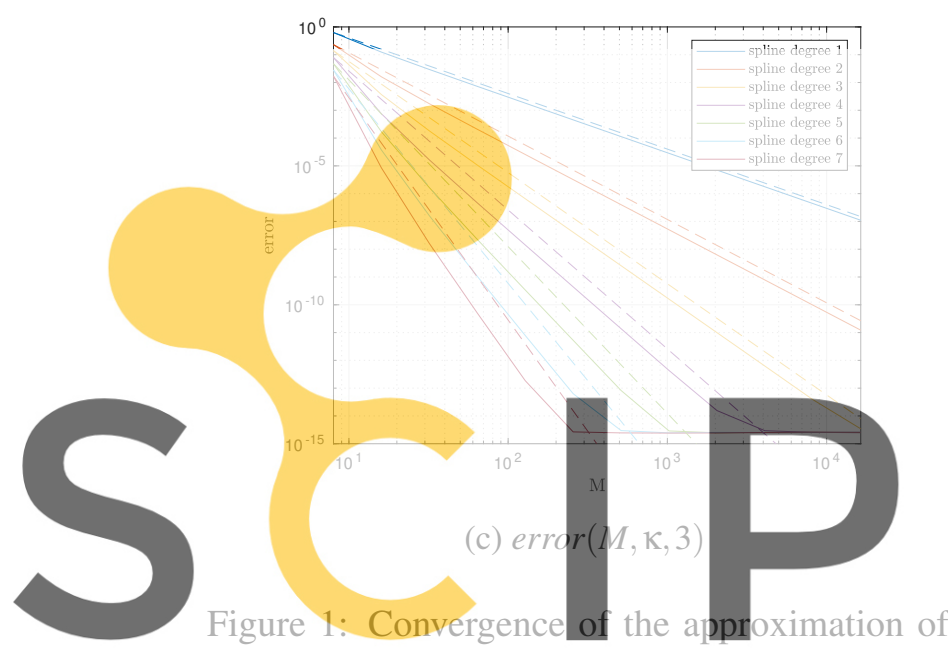

discrete Fourier transform of a spline of various degree $\kappa$ (times the inverse of the Fourier transform of

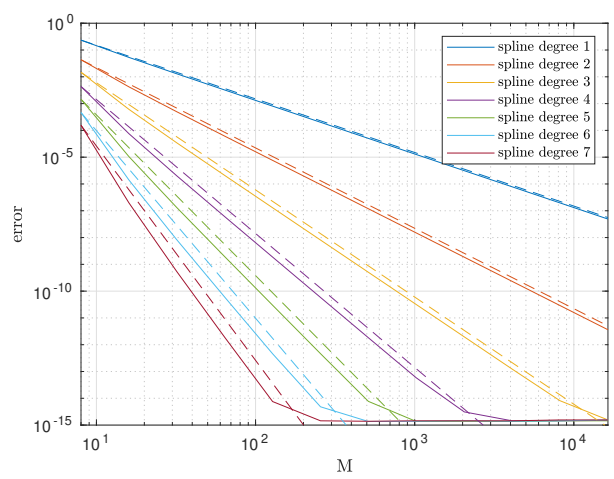

(b) $\operatorname{error}(M, \kappa, 2)$

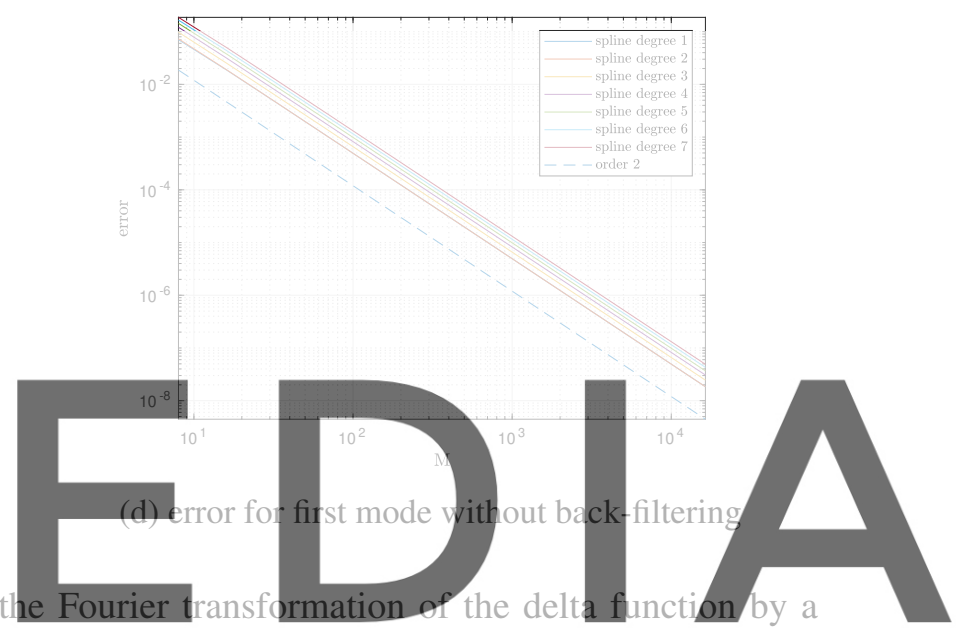

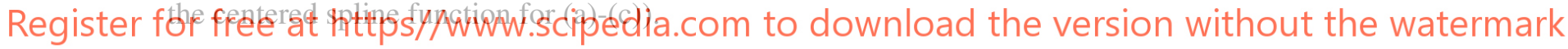

and 30 time units, respectively. We use 10,000 particles for the Weibel test case and 100,000 particles for the two-stream instability. In all cases, we use a Hamiltonian splitting for the time discretization with a fourth order operator splitting and a time step of $\Delta t=0.1$.

In Figure 2 we first compare the time evolutions in the relevant energies (namely, the magnetic energy $\left\|B_{3}\right\|_{2}^{2}$ for the Weibel test case in Panel 2a, and the electric energy $\left\|E_{1}\right\|_{2}^{2}$ for the two-stream instability in Panel 2b. There, the top curves show the time evolution of the energy as computed in a PIF simulation with $K=4$ modes, while the other curves show the errors corresponding with the various PIC simulations, using a fixed grid with $M=64$ points and different spline degrees and back-filtering techniques. As previously explained we have chosen to show the errors corresponding to the variational GEMPIC scheme, but results with the momentum-conserving PIC schemes are similar. From these curves we can see that the error decreases when the degree of the spline is increased for the back-filtered (bff) method, while it remains almost constant for the smoothed Fourier (sf) method, which is in line with our previous convergence results. 
In Figure 3 we next assess some global convergence properties of the back-filtered method as the grid resolution $M$ increases. Panels $3 \mathrm{a}$ and $3 \mathrm{~b}$ show the maximum errors in energy over time (as compared with the PIF solution), whereas Panels $3 \mathrm{c}$ and $3 \mathrm{~d}$ show the momentum conservation errors for the respective time ranges, using B-spline shapes of degree $\kappa$ from 1 to 7 . Overall, these results show that the accuracy increases with the degree of the spline shape function, with convergence rates that follow a $\kappa+1$ trend. Some deviations can be seen however, which are likely due to the nonlinear nature of this convergence experiment. In particular, the convergence region is clearly not reached on the coarse grids for linear splines and there is a roundoff error problem for the finer grids with splines of degree 5 and 6 .

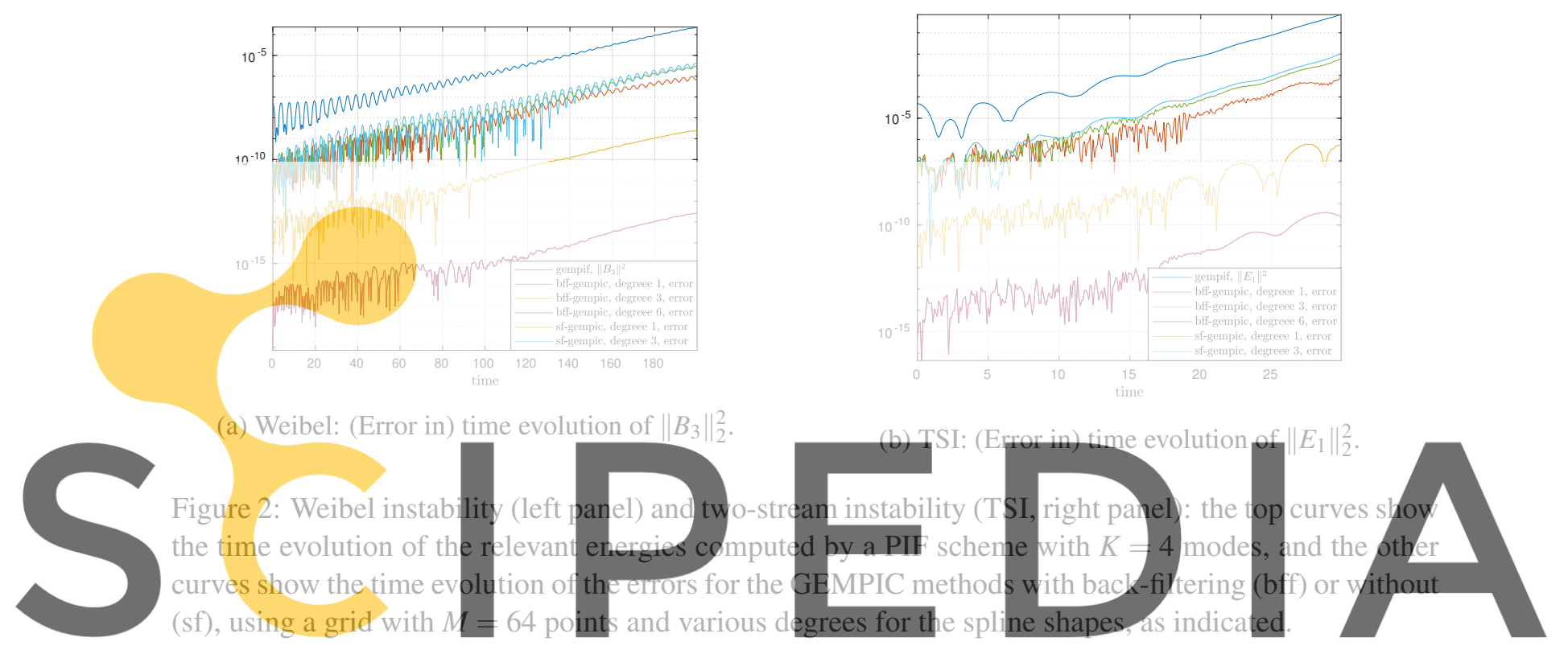

\section{Register for free at https//www.scipedia.com to download the version without the watermark 6 Finite gridl instability}

We end our study with a single-mode electrostatic oscillation problem used in e.g. [3] to investigate the manifestation of finite grid instabilities in spectral PIC codes. Here the plasma consists of a uniform ion background and kinetic electrons with a drift, that initially deviate from the equilibrium density by a small sinusoidal perturbation. For such a problem the system should sustain a stable oscillation of the perturbed mode, but the aliasing errors inherent to the finite grid coupling typically trigger spurious oscillations in PIC simulations. In practice we use a deterministic sampling with particles positions equidistantly distributed in $[0,2 \pi)$ and perturbed with $\delta X_{p}=\varepsilon k^{-1} \cos \left(k X_{p}\right)$, and we set their velocity to $V_{p}=0.01\left(1+k^{-1} \sin \left(k X_{p}\right)\right)$, using a perturbation amplitude $\varepsilon=0.01$ on the mode $k=9$. In all the simulations we use $N \approx 10,000$ particles and a Fourier Maxwell solver with $K=16$ modes. The time scheme is a fourth-order Hamiltonian splitting with $\Delta t=0.1$.

In Figure 4 we show the evolution of the main modes present in the simulations. On the left the PIF simulation exhibits a correct behavior with a regular pulsation of the single mode $k=9$, whereas several spurious modes (namely $k=3,6,12$ and 15) quickly develop in the GEMPIC simulation shown on the 


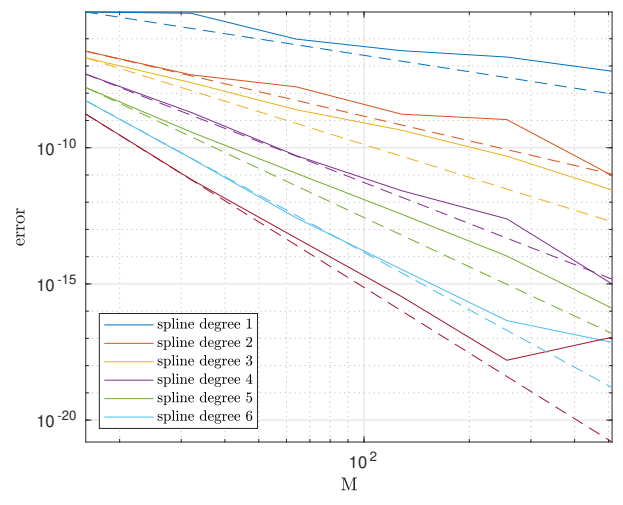

(a) Weibel: Maximum error in $\left\|B_{3}\right\|_{2}^{2}$.
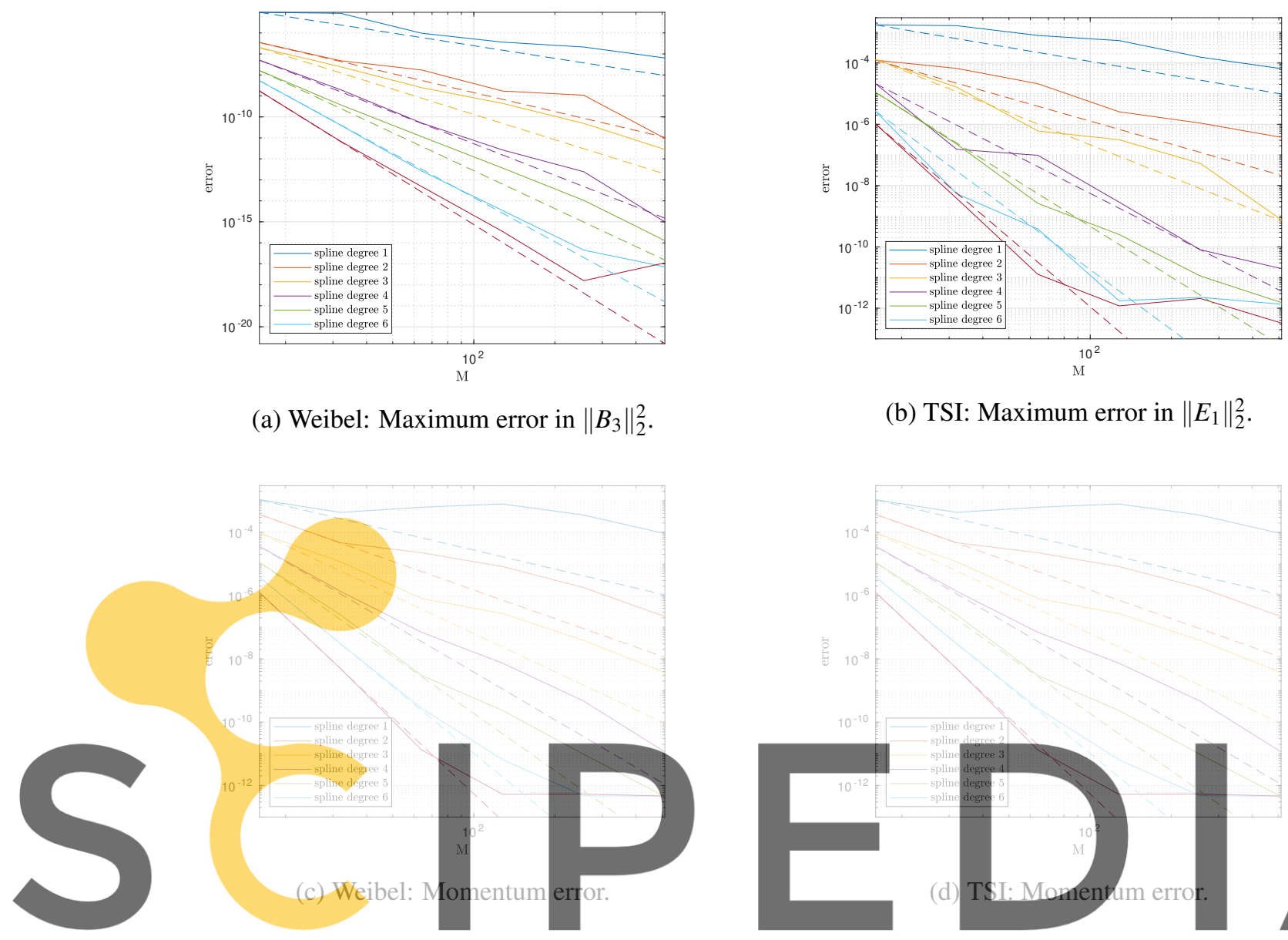

(b) TSI: Maximum error in $\left\|E_{1}\right\|_{2}^{2}$.

Figure 3: Weibel instability (left panels) and two-stream instability (right panels): errors of back-filtered

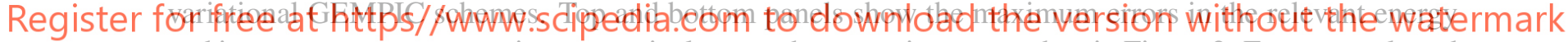
and in momentum conservation respectively, over the same time range than in Figure 2. Errors are plotted versus the number of grid points $M$, using spline shapes of various degrees $\kappa$, as indicated. Dashed lines indicate convergence orders of $\kappa+1$ for the corresponding color.

right, where quadratic splines are used on a grid with $M=33$ points. We note that these spurious modes are mostly triggered by the aliasing of the first harmonic $k=18$ which is outside of the computational range and is folded on the $k=15$ mode by the DFT. This in turn triggers secondary spurious modes by beating with the main one.

In Figures 5 and 6 we then present the results obtained by using spline shapes of higher degrees, namely $\kappa=5$ or 7 , and grids with $M=33$ or 39 points, which corresponds to a very mild oversampling. The runs in Figure 5 have been obtained with a standard spline smoothing, and as expected we see that higher spline degrees reduce the spurious modes to a significantly low level, in particular when a finer grid is used for the DFT. This confirms the well-known anti-aliasing properties of the smooth splines. The interesting observation comes from Figure 6 where an additional Fourier back-filtering (19) has been used 
in the particle-field coupling. There we see that the anti-aliasing effect is less pronounced, in particular for the coarse grid $M=33$. With the finer grid the spurious modes are reduced but slightly less than in Figure 5 (which is not surprising, since back-filtering amplifies the higher modes in the computational range $\llbracket-K, K \rrbracket)$. The main difference lies in the time oscillation of the main mode $k=9$ : whereas it is significantly slowed down when no back-filtering is used, as a consequence of the damping effect applied by the spline smoothing on this mode, we see that in the back-filtered runs it matches almost perfectly with the reference PIF run in Figure 4.
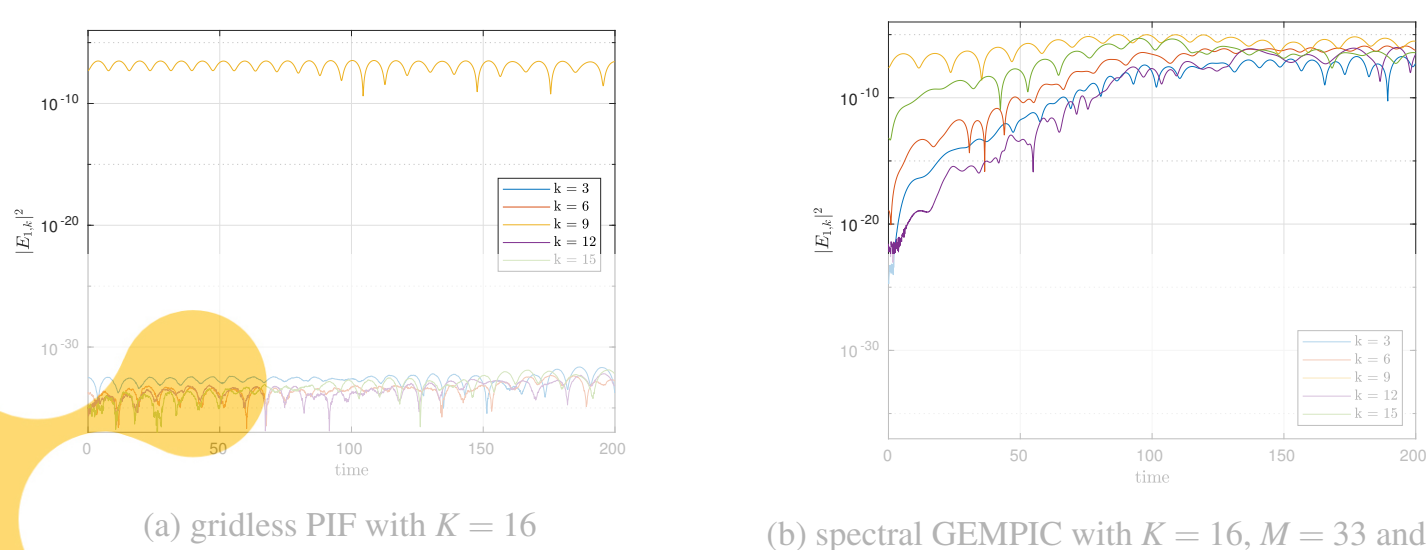

(b) spectral GEMPIC with $K=16, M=33$ and $\kappa=2$

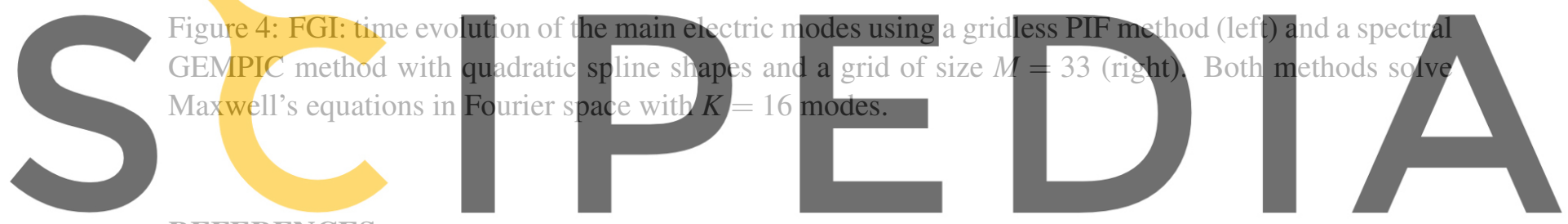

REFERENCES

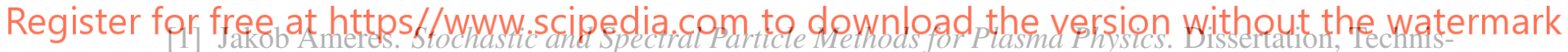
che Universität München, München, 2018.

[2] Martin Campos Pinto, Jakob Ameres, Katharina Kormann, and Eric Sonnendrücker. On Geometric Fourier Particle In Cell Methods. arXiv preprint arXiv:2102.02106, 2021.

[3] C-K Huang, Yong Zeng, Ying Wang, Michael D Meyers, Sunghwan Yi, and Brian J Albright. Finite grid instability and spectral fidelity of the electrostatic particle-in-cell algorithm. Computer Physics Communications, 207:123-135, 2016.

[4] Michael Kraus, Katharina Kormann, Philip J. Morrison, and Eric Sonnendrücker. GEMPIC: Geometric electromagnetic particle-in-cell methods. Journal of Plasma Physics, 83(4), 2017.

[5] A Bruce Langdon. Effects of the spatial grid in simulation plasmas. Journal of Computational Physics, 6(2):247-267, October 1970.

[6] Matthew S Mitchell, Matthew T Miecnikowski, Gregory Beylkin, and Scott E Parker. Efficient Fourier Basis Particle Simulation. Journal of Computational Physics, pages 837-847, August 2019.

[7] N Ohana, A Jocksch, E Lanti, TM Tran, S Brunner, C Gheller, F Hariri, and L Villard. Towards 


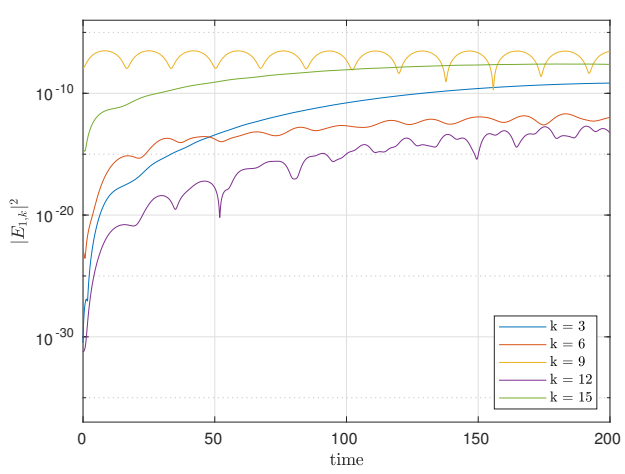

(a) grid $M=33$, degree $\kappa=5$

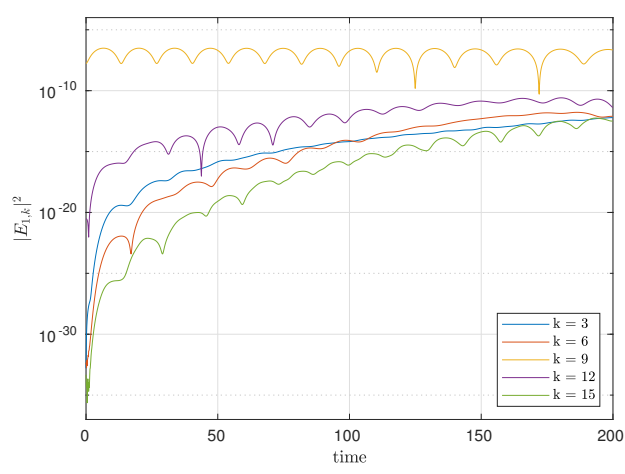

(c) grid $M=39$, degree $\kappa=5$

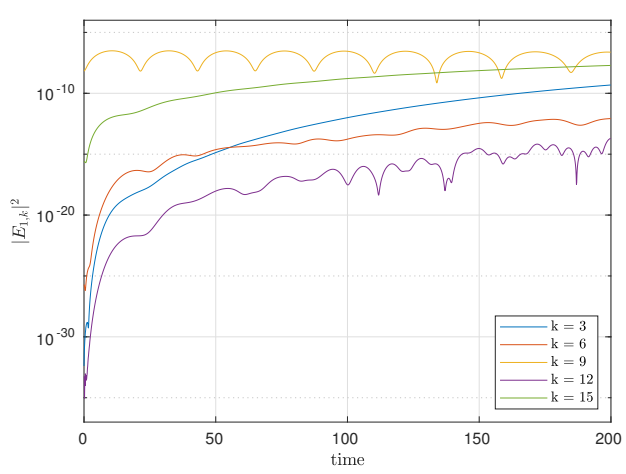

(b) grid $M=33$, degree $\kappa=7$

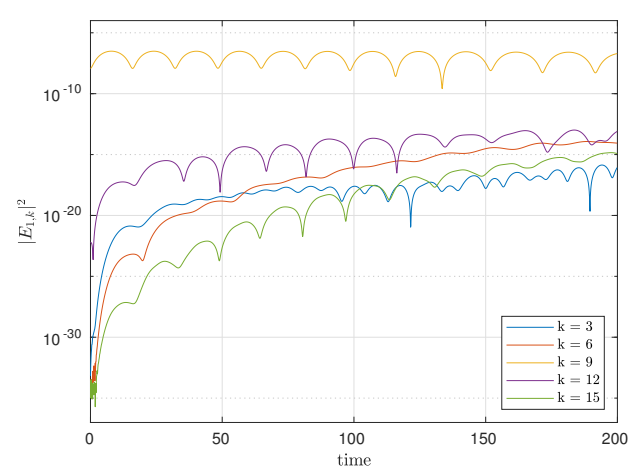

(d) grid $M=39$, degree $\kappa=7$

Figure 5: FGI: time evolution of the main modes using a smoothed Fourier GEMPIC scheme with different grids and shape degrees, and no back-filtering.

the optimization of a gyrokinetic Particle-In-Cell (PIC) code on large-scale hybrid architectures. In Journal of Physics: Conference Series, volume 775, page 012010. IOP Publishing, 2016.

[8] Gerlind Plonka, Daniel Potts, Gabriele Steidl, and Manfred Tasche. Fast Fourier Transforms for Nonequispaced Data. In Numerical Fourier Analysis, pages 377-419. Birkhäuser, Cham, 2018.

[9] Gabriele Steidl. A note on fast Fourier transforms for nonequispaced grids. Advances in Computational Mathematics, 9(3-4):337-352, 1998. 


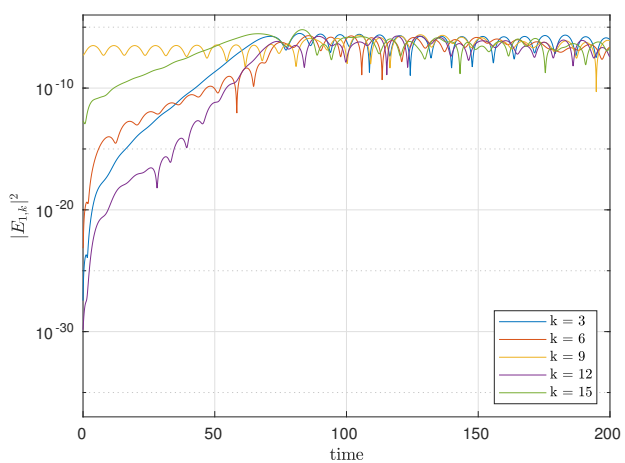

(a) grid $M=33$, degree $\kappa=5$

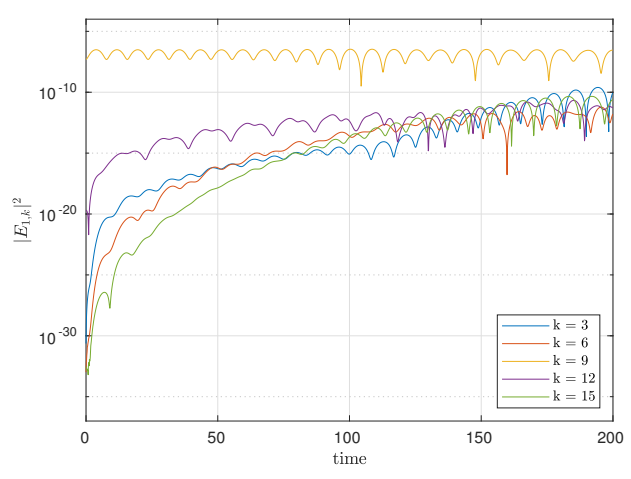

(c) grid $M=39$, degree $\kappa=5$

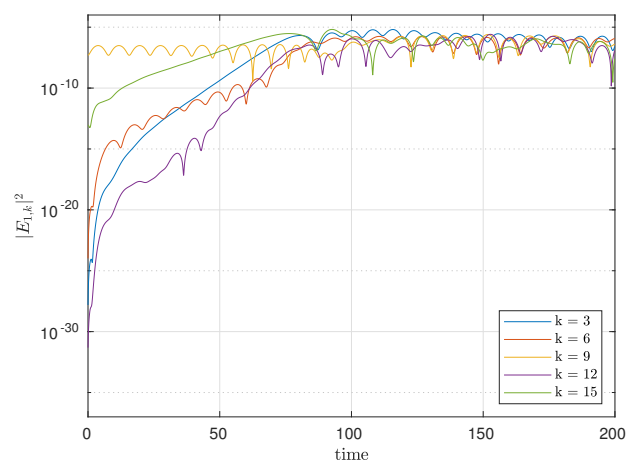

(b) grid $M=33$, degree $\kappa=7$

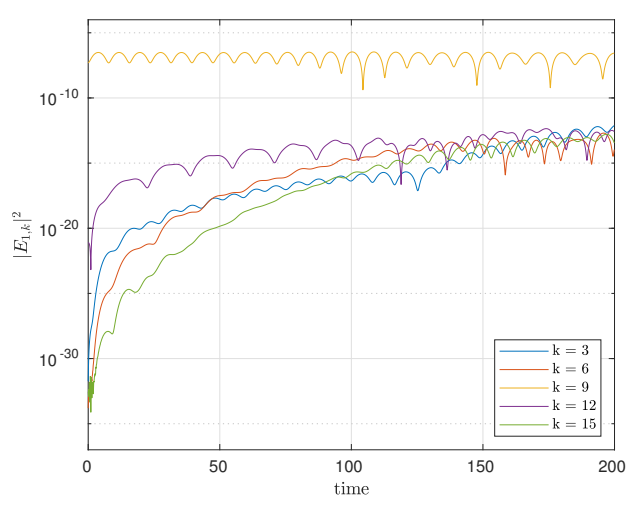

(d) grid $M=39$, degree $\kappa=7$

Figure 6: FGI: time evolution of the main modes using a smoothed Fourier GEMPIC scheme with different grids and shape degrees, and back-filtering. 